\title{
Article \\ Alcohol Drinking Pattern and Risk of Head and Neck Cancer: A Nationwide Cohort Study
}

\author{
Hye Yeon Koo ${ }^{1,+}+$,, Kyungdo Han ${ }^{2,+}$, Dong Wook Shin ${ }^{3,4,5, *}$, Jung Eun Yoo ${ }^{6}$, Mi Hee Cho ${ }^{7}$, Keun Hye Jeon ${ }^{8}$, \\ Dahye Kim ${ }^{9}$, Sangduk Hong ${ }^{10}$ and Jae Kwan Jun ${ }^{11,12}$
}

check for

updates

Citation: Koo, H.Y.; Han, K.; Shin, D.W.; Yoo, J.E.; Cho, M.H.; Jeon, K.H.; Kim, D.; Hong, S.; Jun, J.K. Alcohol Drinking Pattern and Risk of Head and Neck Cancer: A Nationwide Cohort Study. Int. J. Environ. Res. Public Health 2021, 18, 11204. https:// doi.org/10.3390/ijerph182111204

Academic Editor: Marc Saez

Received: 16 September 2021

Accepted: 21 October 2021

Published: 25 October 2021

Publisher's Note: MDPI stays neutral with regard to jurisdictional claims in published maps and institutional affiliations.

Copyright: (c) 2021 by the authors. Licensee MDPI, Basel, Switzerland. This article is an open access article distributed under the terms and conditions of the Creative Commons Attribution (CC BY) license (https:// creativecommons.org/licenses/by/ $4.0 /)$.
1 Health Promotion Center, CHA Bundang Medical Center, Seongnam 13496, Korea; tare4645@chamc.co.kr 2 Department of Statistics and Actuarial Science, Soongsil University, Seoul 06978, Korea; hkd@ssu.ac.kr

3 Department of Family Medicine/Supportive Care Center, Samsung Medical Center, Sungkyunkwan University School of Medicine, Seoul 06351, Korea

4 Department of Digital Health, SAIHST, Sungkyunkwan University, Seoul 06355, Korea

5 Center for Clinical Epidemiology, SAIHST, Sungkyunkwan University, Seoul 06355, Korea

6 Department of Family Medicine, Healthcare System Gangnam Center, Seoul National University Hospital, Seoul 06236, Korea; 83259@snuh.org

7 Samsung C\&T Medical Clinic, Kangbuk Samsung Hospital, Sungkyunkwan University School of Medicine, Seoul 05288, Korea; mh76.cho@samsung.com

8 Department of Family Medicine, CHA Gumi Medical Center, CHA University, Gumi 39295, Korea; kh1228@chamc.co.kr

9 Department of Medical Statistics, The Catholic University of Korea, Seoul 07345, Korea; dhkim373@daewoong.co.kr

10 Department of Otorhinolaryngology, Samsung Medical Center, Sungkyunkwan University School of Medicine, Seoul 06351, Korea; sangduk.hong@samsung.com

11 National Cancer Control Institute, National Cancer Center, Goyang 10408, Korea; jkjun@ncc.re.kr

12 Graduate School of Cancer Science and Policy, National Cancer Center, Goyang 10408, Korea

* Correspondence: dwshin@skku.edu; Tel.: +82-2-6190-5252

+ Contributed equally as first authors.

Abstract: Alcohol consumption is a major risk factor for head and neck cancer (HNC), yet little data exist examining drinking patterns and HNC risk. In this population-based, retrospective cohort study, 11,737,467 subjects were recruited from the Korean National Health Insurance Service database. The risks of overall HNC and HNC subtypes according to average alcohol consumption, drinking frequency, and daily amount were examined using Cox proportional hazard models. Over the median follow-up of 6.4 years, 15,832 HNC cases were identified. HNC risk linearly increased with drinking frequency ( $p$-trend $<0.01$; adjusted hazard ratio [aHR] 1.55, 95\% confidence interval [CI] 1.45-1.67 in subjects who drank 7 days/week). HNC risk also increased according to daily amount of alcohol consumption ( $p$-trend < 0.01), but plateaued from 5-7 units/occasion (aHR 1.25, $95 \%$ CI 1.19-1.31) to $>14$ units/occasion (aHR 1.26, 95\% CI 1.13-1.40). When stratified by average alcohol consumption, drinking frequency, but not daily amount, showed a linear relationship with HNC risk in moderate and heavy drinkers. When comparing the HNC subtypes, similar tendencies were observed in cancers of the oral cavity, pharynx, and larynx, but not in the salivary gland. In conclusion, drinking frequency is a stronger risk factor for $\mathrm{HNC}$, especially for cancer of the oral cavity, pharynx, and larynx, than the daily amount of alcohol consumption.

Keywords: head and neck neoplasms; alcohol drinking; drinking behavior; risk factors; cohort studies

\section{Introduction}

As global cancer incidence and cancer death are rapidly growing, identifying modifiable cancer risk factors is becoming more important [1,2]. There were about 470,000 global death cases from head and neck cancer (HNC)-including cancers of the lip, oral cavity, salivary gland, pharynx, and larynx-in 2020 [1]. Approximately 900,000 patients in the world and 5000 patients in Korea are newly diagnosed with HNC annually [1]. Along with 
cigarette smoking, alcohol consumption is a major independent risk factor for $\mathrm{HNC}$, and reduced exposure to these factors is expected to prevent more than half of the squamous cell carcinomas of the head and neck [3,4].

Previous studies of HNC risk have mostly focused on the average alcohol consumption level [5-8]. These studies, including meta-analyses [7,8], have almost consistently shown that average alcohol intake (g/day) is associated with HNC risk. However, recent reports on several types of cancers, such as breast and prostate cancer, have suggested that drinking patterns, including binge drinking and frequency of drinking, might also affect the carcinogenic effect of alcohol $[9,10]$. In relation to HNC, little data exists on drinking patterns and cancer risk. One study based on two large prospective cohorts observed that the risk of alcohol-related cancers, including cancers of oral cavity, pharynx, larynx, and others, increased with the frequency of drinking in men even when adjusted for total alcohol intake ( $p$ for trend $=0.03$ ) [11]. In another prospective study, the association between the risk of upper aerogastric tract cancers and drinking frequency was examined; however, the daily amount of drinking was not considered [12]. Drinking 4-7 times per week was associated with an increased cancer risk compared to non-drinkers (relative risk [RR] 3.9, 95\% confidence interval [CI] 2.1-7.1) [12]. However, previous cohort studies were based on a limited number of cancer cases (417 [11] and $49 \mathrm{HNC}$ cases [12], respectively), and the risk of various HNC subtypes according to drinking pattern were not examined. In a study by the INHANCE consortium, joint effects of drinking intensity (number of drinks per day) and duration (years) on risks of HNC subtypes were examined [13]. This study has included a relatively large number of HNC cases (4100), yet the analysis was based on case-control results. Risks of all HNC subtypes increased according to alcohol intensity without threshold, whereas risks were not considerably modified by duration except for that of the oropharynx. However, the effect of drinking frequency was not investigated. Another case-control report, which included 240 cancer cases, has observed a linear relationship between drinking frequency and overall upper aerodigestive tract cancers ( $p$ for trend $=0.01$ ) [14], whereas no association was observed between oral cancer subtype $(n=187)$ and drinking frequency in a study based on the same data [15]. There are few meta-analyses or systematic reviews on drinking frequency and overall HNC risk, but one meta-analysis of cohort studies has examined the association between nasopharyngeal cancer risk and drinking frequency [16]. Frequent drinking ( $\geq 7$ times per week) was related to an increase in the risk of nasopharyngeal cancer (pooled odds ratio [OR] 1.29, $95 \%$ CI 1.05-1.53) in this analysis, whereas infrequent drinking ( $<7$ times per week) showed the contrasting result (pooled OR 0.77, 95\% CI 0.60-0.94).

In our study, we aimed to investigate the association between alcohol drinking patterns and the risk of overall $\mathrm{HNC}$ or HNC subtypes using a large population-based cohort. The effects of two components of drinking patterns -drinking frequency and daily amountwere compared using stratified analyses by average alcohol consumption.

\section{Materials and Methods}

\subsection{Study Population and Data Source}

The Korean National Health Insurance Service (NHIS) database was used for this study. The National Health Insurance Service is a mandatory social insurance service managed by the Korean government that provides universal health coverage to nearly the whole population of South Korea [17]. NHIS also provides all subscribers over 40 years old with a national health screening every two years; this program includes assessment of healthrelated behaviors, such as smoking and drinking, as well as anthropometric and laboratory tests. Therefore, the NHIS database contains almost all medical information, including clinical diagnoses, medical procedures, prescription records, and sociodemographic factors, of its beneficiaries.

From this database, $12,724,396$ subjects who were aged over 40 years and who received national health screening in 2009-2010 were initially included. Subjects who were diagnosed with any cancer before the health screening $(n=303,424)$, who had any cancer within 
one year from the health screening $(n=124,472)$, or who were deceased within one year from the health screening $(n=16,182)$ were excluded. Subjects who had any missing data $(n=542,851)$ were also excluded. The final study population comprised $11,737,467$ subjects.

Study subjects were followed from one year after the national health screening date to the date of incident HNC, death, or the last day of the study period (31 December 2017), whichever came first. The median follow-up time was 6.4 years, after a one-year lag period.

\subsection{Ethical Approval}

This study was approved by the Institutional Review Board of Samsung Medical Center (IRB File No. SMC 2019-02-059). The requirement for written informed consent from patients was waived because the data were anonymized under confidentiality guidelines.

\subsection{Exposure to Alcohol}

Data on alcohol consumption was collected from the self-reported questionnaires used for the national health examination in 2009-2010. This questionnaire includes questions about the average drinking frequency (number of days per week) during the last year and the daily amount (standard units per occasion) of drinking, regardless of the type of alcoholic beverage.

Based on this data, we calculated the pure alcohol intake per occasion first. Standard drink size seems to vary among countries, possibly due to cultural differences. [18,19]. One standard unit is commonly defined as $8 \mathrm{~g}$ of pure alcohol in Korea, probably resulting from Korean drinking custom [20]. The majority of alcohol consumed in Korea is beer and Soju (a traditional Korean alcohol beverage), and the amounts of pure alcohol contained in a usual size of cup used for beer $(220 \mathrm{~mL})$ and Soju $(50 \mathrm{~mL})$ in Korea are estimated to be about $8 \mathrm{~g}[20,21]$. Hence, we assumed that one standard unit (one cup of each alcohol beverage type) described in a questionnaire of NHIS contains $8 \mathrm{~g}$ of pure alcohol.

The average weekly alcohol consumption ( $\mathrm{g} /$ week) was calculated by multiplying the pure alcohol intake per occasion and drinking frequency. Since even a lightto-moderate amount of drinking might increase the risk of cancer [11], the average alcohol consumption level was classified in detail as follows: (1) non-drinker (0 g/week); (2) mild drinker (0-105 g/week); (3) moderate drinker (105-210 g/week); and (4) heavy drinker ( $\geq 210 \mathrm{~g} /$ week) [16].

\subsection{Covariates}

Information on potential confounders, including age, sex, income level, smoking status, physical activity, body mass index (BMI), and comorbidities, were collected from the NHIS claims database and the national health screening database. The level of income was categorized into quartiles. Smoking status was categorized into never smoker, ex-smoker, and current smoker; ex-smoker and current smoker were each further categorized into $<20$ pack-years of smoking history and $\geq 20$ pack-years of history. Physical activity level was categorized into non-regular and regular exercise; the regular exercise group was defined as performing either $>30 \mathrm{~min}$ of moderate physical activity at least five times/week or $>20 \mathrm{~min}$ of strenuous physical activity at least three times/week. BMI was calculated by $\mathrm{kg} / \mathrm{m}^{2}$. Baseline comorbidities, including hypertension, diabetes mellitus, and dyslipidemia, were defined based on a combination of health examination records, disease diagnosis codes, and prescription records.

\subsection{Study Outcomes}

Diagnosis of new HNC was the endpoint of this study. Incidence of HNC was identified using claims records with given diagnosis codes, according to the International Classification of Disease, 10th Revision (ICD-10): cancers of the oral cavity (C00-C06), salivary gland (C07, C08), pharynx (C09-C13), larynx (C32), and other sites in the head and neck (C14, C30, C31) were defined as HNC. In addition, a diagnosis of cancer was also identified based on the receipt of a special co-payment reduction program for critical 
illness. In Korea, cancer patients who possess a medical certificate of diagnosis can apply for the special co-payment reduction program. The enrollees of this program pay only $5 \%$ of medical expenses for cancer work-up and cancer treatment, whereas other patients pay usually $20-30 \%$ of expenses for common diseases. Therefore, nearly all cancer patients are registered in this program in Korea, which makes our information on HNC diagnosis extremely reliable.

\subsection{Statistical Analysis}

Descriptive statistics were used to examine the baseline characteristics of the study population according to average alcohol consumption level. A Student's $t$-test was performed to compare continuous variables, and a chi-squared test was performed to compare categorical variables.

Incidence rates of overall $\mathrm{HNC}$ and $\mathrm{HNC}$ subtypes were calculated as the sum of new cases during the follow-up period divided by the sum of the person-years (per 100,000). To evaluate the effect of each independent variable, including average alcohol consumption, drinking frequency, and daily amount, on the risk of developing overall HNC or one of the HNC subtypes, Cox proportional hazard regression analyses were conducted. For all analyses, non-drinkers were used as the reference group. We also adjusted for all of the following covariates in gradual modeling: (1) Model 1 was adjusted for age and sex as a basic analysis; (2) Model 2 was additionally adjusted for variables that generally show associations with HNC or were commonly considered in previous studies, including income, smoking status, physical activity level, BMI and diabetes mellitus [5,14,22,23]; (3) Model 3 was additionally adjusted for common comorbidities which were associated with HNC in a few studies, including hypertension and dyslipidemia [24,25].

The risks of overall HNC and HNC subtypes were stratified by average alcohol consumption level. The impacts of drinking frequency (days per week) and daily amount (standard units per occasion) were examined.

All analyses were performed using SAS version 9.4 (SAS Institute Inc., Cary, NC, USA), and the two-sided significance levels were set at $p<0.05$.

\section{Results}

\subsection{Baseline Demographics}

Table 1 shows the baseline characteristics of the study population. About $40 \%$ of subjects $(n=4,727,135)$ were drinkers. When compared with non-drinkers, drinkers were younger, male, in lower socioeconomic status, and had a smoking history (ex- or current smoker). The proportion of ever-smokers increased with increasing average alcohol consumption level $(p<0.0001)$.

Table 1. Baseline characteristics according to average weekly alcohol consumption.

\begin{tabular}{|c|c|c|c|c|c|c|}
\hline & $\begin{array}{c}\text { Total } \\
(n=11,737,467)\end{array}$ & $\begin{array}{c}\text { Non-Drinker } \\
\quad(0 \mathrm{~g}) \\
(n=7,010,332)\end{array}$ & $\begin{array}{l}\text { Mild Drinker } \\
(0<x<105 \mathrm{~g}) \\
(n=2,781,462)\end{array}$ & $\begin{array}{c}\text { Moderate } \\
\text { Drinker } \\
(105 \leq x<210 \mathrm{~g}) \\
(n=1,113,038)\end{array}$ & $\begin{array}{l}\text { Heavy Drinker } \\
\quad(\geq 210 \mathrm{~g}) \\
(n=832,635)\end{array}$ & $\begin{array}{c}p- \\
\text { Values }\end{array}$ \\
\hline \multicolumn{7}{|c|}{ Mean \pm SD, or No. (\%) } \\
\hline Age (years) & $54.6 \pm 10.4$ & $56.4 \pm 11.0$ & $51.8 \pm 9.7$ & $51.5 \pm 9.1$ & $52.3 \pm 9.5$ & $<0.0001$ \\
\hline Sex (male) & $5,612,691(47.8)$ & $2,027,999(28.9)$ & $1,805,061(64.9)$ & $992,950(89.2)$ & $786,681(94.5)$ & $<0.0001$ \\
\hline $\begin{array}{c}\text { Income (lowest } \\
\text { quartile) }\end{array}$ & $2,985,640(25.4)$ & $1,883,641(26.9)$ & $671,687(24.2)$ & $243,933(21.9)$ & $186,379(22.4)$ & $<0.0001$ \\
\hline Smoking status & & & & & & $<0.0001$ \\
\hline Never smoker & $7,674,834(65.4)$ & $5,810,163(82.9)$ & $1,413,540(50.8)$ & $283,125(25.4)$ & $168,006(20.2)$ & \\
\hline Ex-smoker, <20 PY & $1,064,858(9.1)$ & $330,639(4.7)$ & $426,874(15.4)$ & $190,356(17.1)$ & $116,989(14.1)$ & \\
\hline Ex-smoker, $\geq 20$ PY & $672,441(5.7)$ & $235,408(3.4)$ & $195,990(7.1)$ & $122,703(11.0)$ & $118,340(14.2)$ & \\
\hline $\begin{array}{l}\text { Current smoker, } \\
<20 \mathrm{PY}\end{array}$ & $1,042,913(8.9)$ & $281,892(4.0)$ & $404,652(14.6)$ & $227,469(20.4)$ & $128,900(15.5)$ & \\
\hline
\end{tabular}


Table 1. Baseline characteristics according to average weekly alcohol consumption.

\begin{tabular}{|c|c|c|c|c|c|c|}
\hline & $\begin{array}{c}\text { Total } \\
(n=11,737,467)\end{array}$ & $\begin{array}{c}\text { Non-Drinker } \\
\quad(0 \mathrm{~g}) \\
(n=7,010,332)\end{array}$ & $\begin{array}{l}\text { Mild Drinker } \\
(0<x<105 \mathrm{~g}) \\
(n=2,781,462)\end{array}$ & $\begin{array}{c}\text { Moderate } \\
\text { Drinker } \\
(105 \leq x<210 \mathrm{~g}) \\
(n=1,113,038)\end{array}$ & $\begin{array}{l}\text { Heavy Drinker } \\
\quad(\geq 210 \mathrm{~g}) \\
(n=832,635)\end{array}$ & $\begin{array}{c}p- \\
\text { Values }\end{array}$ \\
\hline \multicolumn{7}{|c|}{ Mean \pm SD, or No. $(\%)$} \\
\hline $\begin{array}{c}\text { Current smoker, } \\
\geq 20 \mathrm{PY}\end{array}$ & $1,282,421(10.9)$ & $352,230(5.0)$ & $340,406(12.2)$ & $289,385(26.0)$ & $300,400(36.1)$ & \\
\hline Physical activity level & & & & & & $<0.0001$ \\
\hline Non-regular & $9,408,663(80.2)$ & $5,736,859(81.8)$ & $2,165,900(77.9)$ & $859,791(77.3)$ & $646,113(77.6)$ & \\
\hline Regular & $2,328,804(19.8)$ & $1,273,473(18.2)$ & $615,562(22.1)$ & $253,247(22.7)$ & $186,522(22.4)$ & \\
\hline $\begin{array}{l}\text { Body mass index } \\
\left(\mathrm{kg} / \mathrm{m}^{2}\right)\end{array}$ & $24.0 \pm 3.0$ & $23.9 \pm 3.1$ & $23.9 \pm 2.9$ & $24.3 \pm 2.9$ & $24.4 \pm 3.0$ & $<0.0001$ \\
\hline $\begin{array}{l}\text { Waist circumference } \\
\qquad(\mathrm{cm})\end{array}$ & $81.1 \pm 8.5$ & $80.0 \pm 8.7$ & $81.5 \pm 8.4$ & $84.1 \pm 7.7$ & $85.1 \pm 7.8$ & $<0.0001$ \\
\hline $\begin{array}{l}\text { Systolic blood pressure } \\
\text { (mmHg) }\end{array}$ & $124.1 \pm 15.4$ & $123.4 \pm 15.7$ & $123.5 \pm 14.9$ & $126.8 \pm 14.8$ & $128.3 \pm 15.1$ & $<0.0001$ \\
\hline $\begin{array}{c}\text { Diastolic blood } \\
\text { pressure }(\mathrm{mmHg})\end{array}$ & $77.0 \pm 10.1$ & $76.1 \pm 10.1$ & $77.2 \pm 10.1$ & $79.6 \pm 10.0$ & $80.5 \pm 10.1$ & $<0.0001$ \\
\hline $\begin{array}{l}\text { Fasting glucose } \\
(\mathrm{mg} / \mathrm{dL})\end{array}$ & $99.9 \pm 24.5$ & $98.9 \pm 24.0$ & $99.4 \pm 23.4$ & $103.0 \pm 26.5$ & $105.8 \pm 29.2$ & $<0.0001$ \\
\hline $\begin{array}{l}\text { Total cholesterol } \\
(\mathrm{mg} / \mathrm{dL})\end{array}$ & $199.2 \pm 37.0$ & $199.8 \pm 37.6$ & $198.1 \pm 35.8$ & $199.0 \pm 36.1$ & $198.4 \pm 37.0$ & $<0.0001$ \\
\hline HDL (mg/dL) & $54.8 \pm 16.7$ & $54.5 \pm 17.3$ & $55.0 \pm 15.4$ & $55.1 \pm 15.5$ & $56.1 \pm 16.6$ & $<0.0001$ \\
\hline $\mathrm{LDL}(\mathrm{mg} / \mathrm{dL})$ & $117.4 \pm 34.1$ & $119.9 \pm 34.3$ & $116.1 \pm 33.1$ & $111.9 \pm 34.2$ & $107.7 \pm 35.3$ & $<0.0001$ \\
\hline $\begin{array}{c}\text { eGFR } \\
\left(\mathrm{ml} / \mathrm{min} / 1.73 \mathrm{~m}^{2}\right)\end{array}$ & $85.8 \pm 34.5$ & $84.6 \pm 31.0$ & $86.7 \pm 38.1$ & $88.3 \pm 41.0$ & $89.7 \pm 40.4$ & $<0.0001$ \\
\hline Hypertension & $3,975,024(33.9)$ & $2,406,414(34.3)$ & $829,585(29.8)$ & $404,736(36.4)$ & $334,289(40.2)$ & $<0.0001$ \\
\hline Diabetes mellitus & $1,374,937(11.7)$ & $832,222(11.9)$ & $274,386(9.9)$ & $141,392(12.7)$ & $126,937(15.3)$ & $<0.0001$ \\
\hline Dyslipidemia & $2,616,859(22.3)$ & $1,815,516(25.9)$ & $564,073(20.3)$ & $237,270(21.3)$ & $181,905(21.9)$ & $<0.0001$ \\
\hline
\end{tabular}

Abbreviations: SD, standard deviation; PY, pack-years; eGFR, estimated glomerular filtration rate; HDL, high-density lipoprotein cholesterol; LDL, low-density lipoprotein cholesterol.

\subsection{Risk of HNC According to Drinking Status}

$15,832 \mathrm{HNC}$ cases were identified during the follow-up period. The risk of developing $\mathrm{HNC}$ according to average alcohol consumption, drinking frequency (days per week), and daily amount (standard units per occasion) are presented in Table 2 and Figure 1. When comparing the effects of average alcohol consumption on HNC risk, mild drinkers showed no significant difference in HNC risk compared to non-drinkers, but moderate and heavy drinkers had a higher risk of HNC (adjusted hazard ratio [aHR] 1.26, 95\% CI 1.20-1.32 and aHR 1.46, 95\% CI 1.38-1.53, respectively, in Model 3). As for drinking frequency, HNC risk increased almost linearly in a dose-response manner ( $p$-trend $<0.01)$ : the risk began to significantly increase in subjects who drank 2 days/week (aHR 1.10, 95\% CI 1.05-1.16) compared to non-drinkers and was highest in subjects who drank 7 days/week (aHR 1.55, $95 \%$ CI 1.45-1.67). HNC risk was also increased according to the daily amount per occasion ( $p$-trend $<0.01$ ): it continuously increased in subjects who drank $\leq 5-7$ standard units per occasion when compared to non-drinkers, and then plateaued afterwards (aHR 1.26, 95\% CI 1.13-1.40 in subjects who drank $>14$ units/occasion). Regarding the adjustment model, the addition of comorbidities of hypertension and dyslipidemia (Model 3) did not produce a substantially different result from that of Model 2. 
Table 2. Risk of overall head \& neck cancer according to drinking status.

\begin{tabular}{|c|c|c|c|c|c|c|c|}
\hline & No. $(\%)$ & $\begin{array}{c}\text { Event } \\
\text { Num- } \\
\text { ber }\end{array}$ & $\begin{array}{l}\text { Person-Years } \\
\text { (PYs) }\end{array}$ & $\begin{array}{l}\text { Incidence Rate } \\
\text { (Per 100,000 PYs) }\end{array}$ & $\begin{array}{c}\text { Model } 1 \\
\text { HR (95\% CI) }\end{array}$ & $\begin{array}{c}\text { Model } 2 \\
\text { HR (95\% CI) }\end{array}$ & $\begin{array}{c}\text { Model } 3 \\
\text { HR (95\% CI) }\end{array}$ \\
\hline \multicolumn{8}{|c|}{ Average alcohol consumption ${ }^{a}$} \\
\hline Non-drinker & $7,010,332(59.7)$ & 7503 & $47,804,729.9$ & 15.7 & 1 (ref.) & 1 (ref.) & 1 (ref.) \\
\hline Mild drinker & $2,781,462(23.7)$ & 3746 & $19,061,909.8$ & 19.7 & $\begin{array}{c}1.06 \\
(1.02-1.11)\end{array}$ & $\begin{array}{c}1.03 \\
(0.99-1.07)\end{array}$ & $\begin{array}{c}1.03 \\
(0.98-1.07)\end{array}$ \\
\hline $\begin{array}{l}\text { Moderate } \\
\text { drinker }\end{array}$ & $1,113,038(9.5)$ & 2299 & $7,606,759.9$ & 30.2 & $\begin{array}{c}1.42 \\
(1.36-1.50)\end{array}$ & $\begin{array}{c}1.27 \\
(1.20-1.33)\end{array}$ & $\begin{array}{c}1.26 \\
(1.20-1.32)\end{array}$ \\
\hline $\begin{array}{l}\text { Heavy } \\
\text { drinker }\end{array}$ & $832,635(7.1)$ & 2284 & $5,658,733.2$ & 40.4 & $\begin{array}{c}1.74 \\
(1.65-1.83)\end{array}$ & $\begin{array}{c}1.47 \\
(1.39-1.55)\end{array}$ & $\begin{array}{c}1.46 \\
(1.38-1.53)\end{array}$ \\
\hline$p$-trend & & & & & $<0.01$ & $<0.01$ & $<0.01$ \\
\hline \multicolumn{8}{|c|}{ Drinking frequency (days per week) } \\
\hline 0 & $7,010,332(59.7)$ & 7503 & $47,804,729.9$ & 15.7 & 1 (ref.) & 1 (ref.) & 1 (ref.) \\
\hline 1 & $1,897,327(16.2)$ & 2067 & $13,038,365.9$ & 15.9 & $\begin{array}{c}0.94 \\
(0.90-0.99)\end{array}$ & $\begin{array}{c}0.92 \\
(0.88-0.97)\end{array}$ & $\begin{array}{c}0.92 \\
(0.88-0.97)\end{array}$ \\
\hline 2 & $1,224,552(10.4)$ & 1947 & $8,395,959.4$ & 23.2 & $\begin{array}{c}1.18 \\
(1.12-1.25)\end{array}$ & $\begin{array}{c}1.11 \\
(1.05-1.17)\end{array}$ & $\begin{array}{c}1.10 \\
(1.05-1.16)\end{array}$ \\
\hline 3 & $787,753(6.7)$ & 1675 & $5,376,661.1$ & 31.2 & $\begin{array}{c}1.43 \\
(1.36-1.52)\end{array}$ & $\begin{array}{c}1.29 \\
(1.22-1.36)\end{array}$ & $\begin{array}{c}1.28 \\
(1.21-1.36)\end{array}$ \\
\hline 4 & $272,126(2.3)$ & 698 & $1,852,052.9$ & 37.7 & $\begin{array}{c}1.63 \\
(1.50-1.76)\end{array}$ & $\begin{array}{c}1.42 \\
(1.31-1.54)\end{array}$ & $\begin{array}{c}1.41 \\
(1.30-1.53)\end{array}$ \\
\hline 5 & $208,885(1.8)$ & 575 & $1,415,586$ & 40.6 & $\begin{array}{c}1.62 \\
(1.48-1.76)\end{array}$ & $\begin{array}{c}1.39 \\
(1.27-1.52)\end{array}$ & $\begin{array}{c}1.38 \\
(1.26-1.50)\end{array}$ \\
\hline 6 & $117,079(1.0)$ & 420 & $787,973.5$ & 53.3 & $\begin{array}{c}1.79 \\
(1.62-1.98)\end{array}$ & $\begin{array}{c}1.51 \\
(1.37-1.67)\end{array}$ & $\begin{array}{c}1.50 \\
(1.36-1.66)\end{array}$ \\
\hline 7 & 219,413 (1.9) & 947 & $1,460,804.3$ & 64.8 & $\begin{array}{c}1.85 \\
(1.72-1.98)\end{array}$ & $\begin{array}{c}1.56 \\
(1.45-1.67)\end{array}$ & $\begin{array}{c}1.55 \\
(1.45-1.67)\end{array}$ \\
\hline$p$-trend & & & & & $<0.01$ & $<0.01$ & $<0.01$ \\
\hline \multicolumn{8}{|c|}{ Daily amount (standard units per occasion) } \\
\hline 0 & $7,010,332(59.7)$ & 7503 & $47,804,729.9$ & 15.7 & 1 (ref.) & 1 (ref.) & 1 (ref.) \\
\hline $1-2$ & $827,080(7.0)$ & 1055 & $5,641,265.8$ & 18.7 & $\begin{array}{c}0.99 \\
(0.92-1.05)\end{array}$ & $\begin{array}{c}0.98 \\
(0.92-1.05)\end{array}$ & $\begin{array}{c}0.98 \\
(0.92-1.05)\end{array}$ \\
\hline $3-4$ & $1,155,831(9.8)$ & 2006 & $7,906,930.4$ & 25.4 & $\begin{array}{c}1.22 \\
(1.16-1.29)\end{array}$ & $\begin{array}{c}1.15 \\
(1.10-1.21)\end{array}$ & $\begin{array}{c}1.15 \\
(1.09-1.21)\end{array}$ \\
\hline $5-7$ & $1,622,238(13.8)$ & 3240 & $11,084,760.3$ & 29.2 & $\begin{array}{c}1.41 \\
(1.35-1.48)\end{array}$ & $\begin{array}{c}1.26 \\
(1.20-1.32)\end{array}$ & $\begin{array}{c}1.25 \\
(1.19-1.31)\end{array}$ \\
\hline 8-14 & $923,266(7.9)$ & 1668 & $6,330,255.9$ & 26.4 & $\begin{array}{c}1.43 \\
(1.35-1.51)\end{array}$ & $\begin{array}{c}1.25 \\
(1.18-1.32)\end{array}$ & $\begin{array}{c}1.24 \\
(1.17-1.31)\end{array}$ \\
\hline$>14$ & $198,720(1.7)$ & 360 & $1,364,190.6$ & 26.4 & $\begin{array}{c}1.47 \\
(1.32-1.64)\end{array}$ & $\begin{array}{c}1.27 \\
(1.14-1.41)\end{array}$ & $\begin{array}{c}1.26 \\
(1.13-1.40)\end{array}$ \\
\hline$p$-trend & & & & & $<0.01$ & $<0.01$ & $<0.01$ \\
\hline
\end{tabular}

a Average weekly alcohol consumption level: (1) none (0 g); (2) mild (0-105 g); (3) moderate (105-210 g); (4) heavy ( $\geq 210 \mathrm{~g})$. Model 1: adjusted for age and sex. Model 2: adjusted for age, sex, income, smoking status, physical activity, body mass index, and diabetes mellitus. Model 3: adjusted for age, sex, income, smoking status, physical activity, body mass index, diabetes mellitus, hypertension, and dyslipidemia (The categories for the individual covariates were those defined in Table 1). Abbreviations: HR, hazard ratio; $\mathrm{CI}$, confidence interval. Bold font indicates statistical significance. 

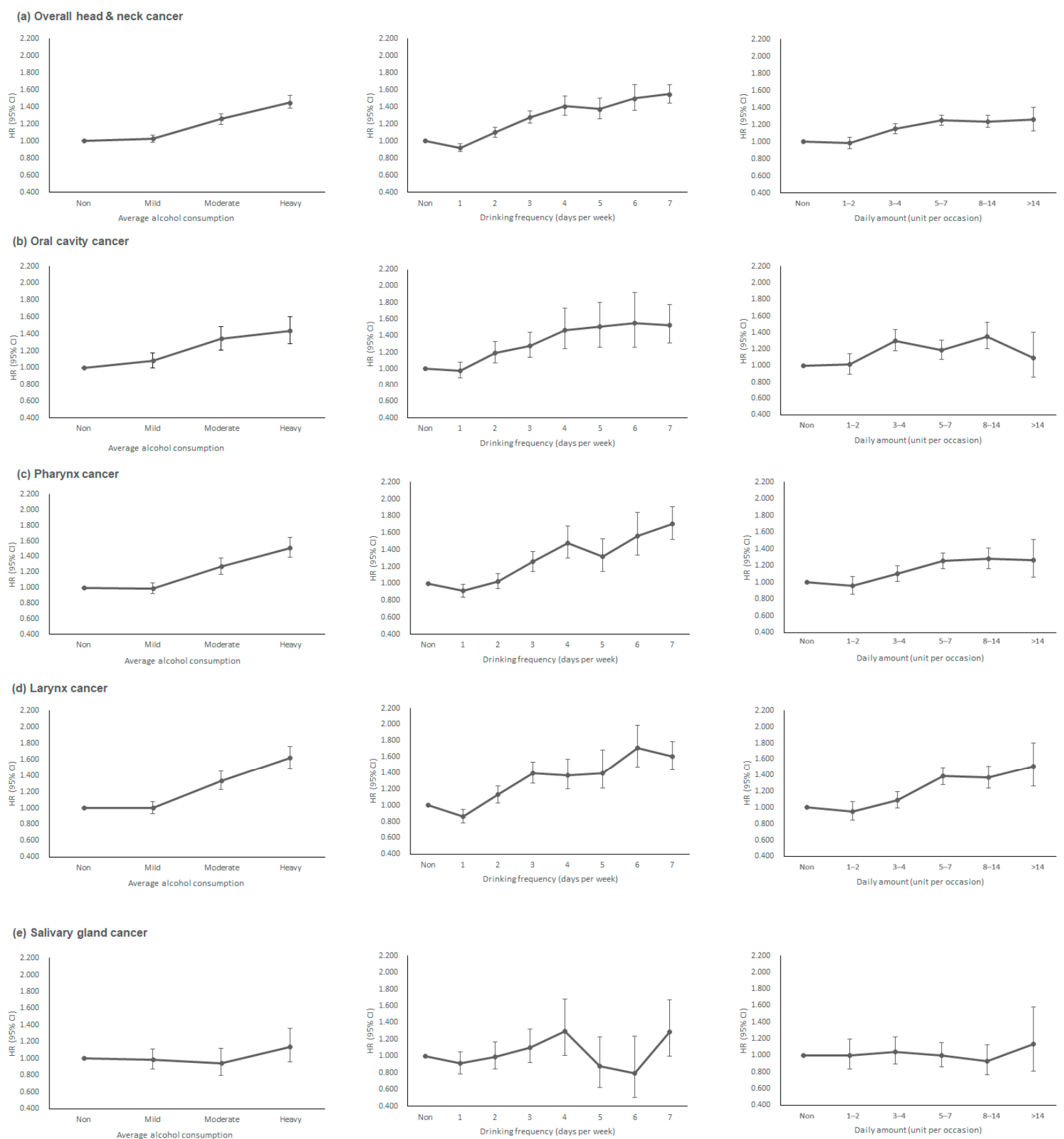

Figure 1. Risk of head \& neck cancer according to alcohol drinking status. HR, hazard ratio; CI, confidence interval. HRs are adjusted for age, sex, income, smoking status, physical activity, body mass index, diabetes mellitus, hypertension and dyslipidemia. (a) overall head \& neck cancer, (b) oral cavity cancer, (c) pharynx cancer, (d) larynx cancer, (e) salivary gland cancer.

The results of analyses on HNC subtypes and drinking status are presented in Table 3 and Figure 1. Cancers of the oral cavity and pharynx cancers showed similar results to those associated with overall HNC risk; risks of these cancers increased almost linearly as average alcohol consumption and drinking frequency increased (aHR 1.44, 95\% CI 1.29-1.60, and aHR 1.51, 95\% CI 1.39-1.64, respectively, in heavy drinkers; aHR 1.53, 95\% CI 1.31-1.78, and aHR $1.71,95 \%$ CI 1.53-1.91, respectively, in subjects who drank 7 days/week). Risks of these cancers were also increased in subjects who drank more than 3-4 standard units per occasion (aHR 1.30, 95\% CI 1.18-1.45, and aHR 1.10, 95\% CI 1.01-1.20, respectively, in subjects who drank 3-4 units/occasion), but this increased risk was less prominent 
with higher daily amounts of alcohol. In the case of larynx cancer, cancer risk increased almost linearly according to average alcohol consumption, drinking frequency, and daily amount (aHR 1.62, 95\% CI 1.49-1.76 in heavy drinkers; aHR 1.61, 95\% CI 1.44-1.79 in subjects who drank 7 days/week; aHR 1.51, 95\% CI 1.27-1.80 in subjects who drank > 14 units/day). No definite tendency was observed for the risk of salivary gland cancer according to drinking status.

Table 3. Risks of head \& neck cancer subtypes according to drinking status.

\begin{tabular}{|c|c|c|c|c|}
\hline & \multicolumn{4}{|c|}{ Cancer Subtypes } \\
\hline & $\begin{array}{c}\text { Oral Cavity } \\
\text { HR }(95 \% \text { CI })\end{array}$ & $\begin{array}{c}\text { Pharynx } \\
\text { HR (95\% CI) }\end{array}$ & $\begin{array}{c}\text { Larynx } \\
\text { HR }(95 \% \text { CI })\end{array}$ & $\begin{array}{c}\text { Salivary Gland } \\
\text { HR }(95 \% \text { CI })\end{array}$ \\
\hline Event $\mathrm{N}$ & 4275 & 5598 & 4862 & 1916 \\
\hline \multicolumn{5}{|c|}{ Average alcohol consumption ${ }^{a}$} \\
\hline Non-drinker & 1 (ref.) & 1 (ref.) & 1 (ref.) & 1 (ref.) \\
\hline Mild drinker & $1.08(1.00-1.17)$ & $0.98(0.92-1.06)$ & $1.00(0.93-1.08)$ & $0.99(0.88-1.11)$ \\
\hline Moderate drinker & $1.34(1.21-1.49)$ & $1.27(1.17-1.38)$ & $1.33(1.22-1.45)$ & $0.95(0.80-1.12)$ \\
\hline Heavy drinker & $1.44(1.29-1.60)$ & $1.51(1.39-1.64)$ & $1.62(1.49-1.76)$ & $1.14(0.96-1.36)$ \\
\hline \multicolumn{5}{|c|}{ Drinking frequency (days per week) } \\
\hline 0 & 1(ref.) & 1 (ref.) & 1 (ref.) & 1 (ref.) \\
\hline 1 & $0.98(0.89-1.08)$ & $0.91(0.84-1.00)$ & $0.86(0.79-0.95)$ & $0.91(0.79-1.05)$ \\
\hline 2 & $1.19(1.07-1.33)$ & $1.03(0.94-1.12)$ & $1.13(1.03-1.24)$ & $0.99(0.85-1.17)$ \\
\hline 3 & $1.28(1.14-1.44)$ & $1.26(1.15-1.38)$ & $1.40(1.27-1.54)$ & $1.10(0.92-1.32)$ \\
\hline 4 & $1.47(1.24-1.73)$ & $1.48(1.30-1.68)$ & $1.37(1.20-1.57)$ & $1.30(1.01-1.68)$ \\
\hline 5 & $1.51(1.26-1.80)$ & $1.32(1.14-1.53)$ & $1.40(1.21-1.61)$ & $0.88(0.63-1.23)$ \\
\hline 6 & $1.56(1.26-1.92)$ & $1.57(1.33-1.84)$ & $1.71(1.48-1.99)$ & $0.79(0.51-1.24)$ \\
\hline 7 & $1.53(1.31-1.78)$ & $1.71(1.53-1.91)$ & $1.61(1.44-1.79)$ & $1.29(1.00-1.67)$ \\
\hline \multicolumn{5}{|c|}{ Daily amount (standard units per occasion) } \\
\hline 0 & 1 (ref.) & 1 (ref.) & 1 (ref.) & 1 (ref.) \\
\hline $1-2$ & $1.02(0.89-1.15)$ & $0.96(0.86-1.07)$ & $0.95(0.85-1.07)$ & $1.00(0.83-1.20)$ \\
\hline $3-4$ & $1.30(1.18-1.45)$ & $1.10(1.01-1.20)$ & 1.09 (0.99-1.19) & $1.04(0.89-1.22)$ \\
\hline $5-7$ & $1.19(1.08-1.31)$ & $1.26(1.17-1.35)$ & 1.39 (1.29-1.50) & $1.00(0.86-1.15)$ \\
\hline $8-14$ & $1.35(1.20-1.52)$ & $1.28(1.17-1.41)$ & $1.37(1.24-1.51)$ & $0.93(0.77-1.13)$ \\
\hline$>14$ & $1.10(0.86-1.40)$ & $1.27(1.06-1.51)$ & $1.51(1.27-1.80)$ & $1.13(0.81-1.58)$ \\
\hline
\end{tabular}

${ }^{a}$ Average weekly alcohol consumption level: (1) none (0 g); (2) mild (0-105 g); (3) moderate (105-210 g); (4) heavy ( $\geq 210$ g). Hazard ratios are adjusted for age, sex, income, smoking status, physical activity, body mass index, diabetes mellitus, hypertension, and dyslipidemia. Abbreviations: $\mathrm{HR}$, hazard ratio; CI, confidence interval. Bold font indicates statistical significance.

\subsection{Risk of HNC and Drinking Pattern: Drinking Frequency vs. Daily Amount}

Supplementary Table S1 and Figure 2 show the risk of overall HNC stratified by average alcohol consumption. In the mild drinker group, no definite trend was observed according to either drinking frequency or daily amount. For drinking frequency, the risk of HNC was highest among subjects who drank 3-4 days/week (aHR 1.29, 95\% CI 1.18-1.40 in Model 2) in mild drinkers. As for the daily amount, $\mathrm{HNC}$ risk was highest in subjects who drank 3-4 units/occasion (aHR 1.10, 95\% CI 1.04-1.17 in Model 2).

Moderate drinkers showed a nearly linear increase in HNC risk according to drinking frequency (aHR 1.32, 95\% CI 1.19-1.46 in subjects who drank 5-7 days/week), whereas HNC risk decreased with increasing daily amount among subjects who drank at least 3-4 units/occasion.

In heavy drinkers, HNC risk linearly increased according to drinking frequency (aHR 1.60, 95\% CI 1.51-1.70 in subjects who drank 5-7 days/week), whereas no definite difference in HNC risk was observed according to the daily amount.

When comparing the associations between drinking patterns and risks of developing one of the HNC subtypes, similar tendencies were observed in cancers of the oral cavity, pharynx, and larynx, but not salivary gland cancers (Supplementary Table S2 and Figure 2). 


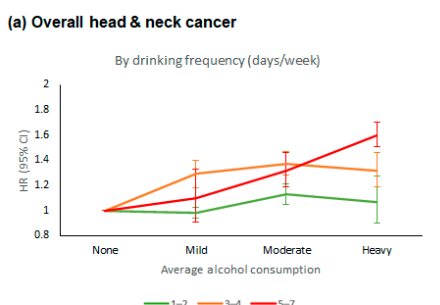

${ }^{1-2}-3-4-5-7$

(b) Oral cavity cancer

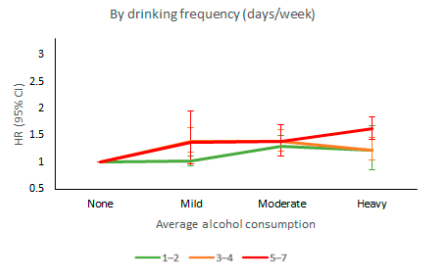

(c) Pharynx cancer

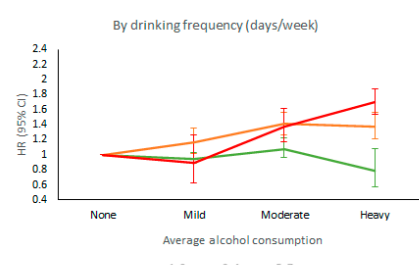

(d) Larynx cancer

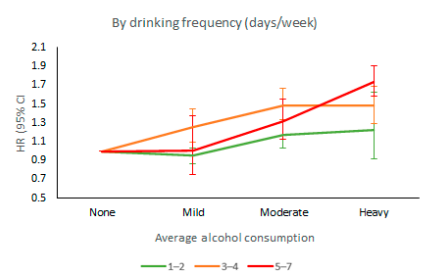

(e) Salivary gland cancer

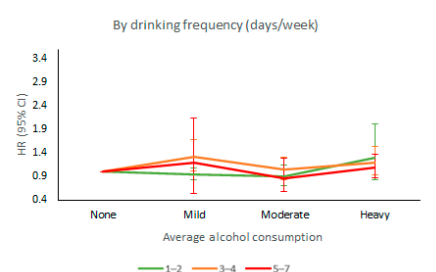

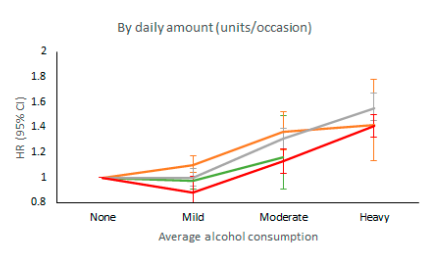

$-^{1-2}-^{3-4} \longrightarrow^{5-7} \longrightarrow 28$
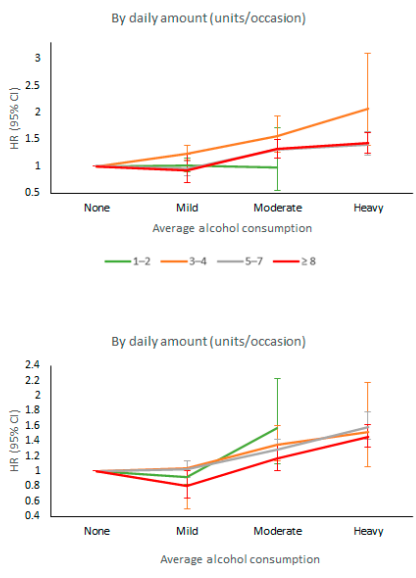

$-^{1-2}-^{3-4}-^{5-7} \longrightarrow_{28}^{28}$

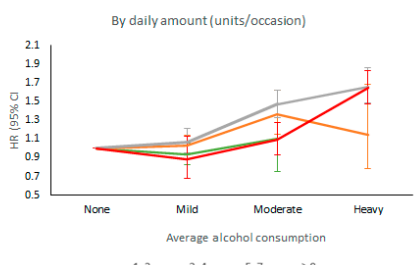

$-1-2-3-4-5-7-3$

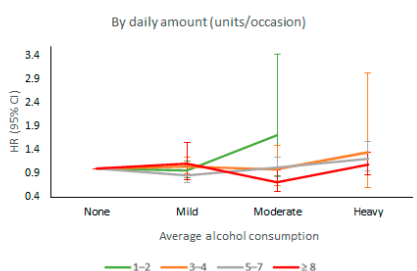

Figure 2. Risk of head \& neck cancer stratified by average alcohol consumption: Impact of drinking frequency versus daily amount. (a) overall head \& neck cancer, (b) oral cavity cancer, (c) pharynx cancer, (d) larynx cancer, (e) salivary gland cancer. HR, hazard ratio; CI, confidence interval. HRs are adjusted for age, sex, income, smoking status, physical activity, body mass index, and diabetes mellitus.

\section{Discussion}

In this large-scale, nationwide, cohort study, average alcohol consumption was associated with an increased risk of overall HNC. The frequency of drinking was almost linearly associated with the risk of overall HNC. The daily amount of drinking also showed a positive association with overall $\mathrm{HNC}$ risk, but the level of association was weaker than that observed with drinking frequency. When comparing HNC subtypes, cancers of the oral cavity, pharynx, and larynx showed similar patterns as those of overall HNC, but salivary gland cancer showed no definite tendency according to drinking status.

We found that average alcohol consumption was positively associated with HNC risk, which is consistent with previous research. Numerous studies have reported that drinking is an independent risk factor of $\mathrm{HNC}$ and that average alcohol consumption level is strongly 
associated with HNC risk in a dose-response manner [5,7,14,26-28]. The carcinogenic effect of alcohol is speculated to mainly result from acetaldehyde (AA), which is the metabolite generated from ethanol $[29,30]$. Suggested mechanisms of AA-related cancer development include interruption of the copying of DNA during cell division, interference in DNA repair, and induction of mutations by forming DNA adducts [29,31]. Recent studies have reported that ethanol metabolism can occur in the oral cavity independently from the liver [32,33], which is in line with findings that AA has been observed in saliva in higher levels than those found in blood, and salivary AA concentration increases dose-dependently according to alcohol consumption $[29,34]$. Hence, salivary AA is thought to contribute to direct carcinogenesis in the oral cavity and pharynx $[29,35]$.

When comparing HNC subtypes, associations of similar magnitudes were observed in cancers of the oral cavity, pharynx, and larynx ( $\mathrm{aHR}=1.44,1.51$, and 1.62 in heavy drinkers, respectively) in our study. Results regarding the differential strength of associations between HNC subtypes and alcohol consumption have varied between studies $[4,6,7,26]$. A meta-analysis of $14,000 \mathrm{HNC}$ patients found that the association between drinking and cancer risk was strongest in pharynx cancer; pooled OR and 95\% CIs in the heavy drinking group were 5.70 (3.61-9.02), 3.93 (2.78-5.57), and 3.00 (1.76-5.11) for pharynx, oral cavity, and larynx cancer, respectively [7]. However, that study was based purely on case-control results, so recall bias might have led to overestimated associations between drinking and HNC risk. On the other hand, a prospective case-cohort study observed that oral cavity cancer was most strongly related to alcohol consumption, followed by pharynx cancer and larynx cancer $(\mathrm{RR}=6.39,3.52$, and 1.54 in subjects drinking $\geq 30 \mathrm{~g}$ ethanol/day, respectively) [26]. However, that study included a limited number of HNC cases (395 overall HNC cases), far fewer than that of our study, potentially lowering their statistical power. Therefore, our results, based on a large, population-based cohort, may be much more reliable than previous results. However, unlike our study, both of the previous studies reported that larynx cancer was least associated with alcohol consumption, which the authors hypothesized might be the result of the larynx having less direct contact with alcohol than the oral cavity or pharynx $[7,26]$. One possible explanation for this observed difference is ethnicity; the Korean population tends to show a lower HNC incidence rate when compared with non-Hispanic white or non-Hispanic black populations, and the prevalence of each HNC subtype is also different among these populations [36]. Considering that most of the previous studies were based on Caucasian populations [7,26], ethnic differences might have resulted in weaker associations between HNC and drinking, and masked differences between HNC subtypes.

The risk of salivary gland cancer showed no definite association with alcohol consumption in our study. Because of its rarity [1], little is known about the risk factors of salivary gland cancer. However, most of the previous investigations on drinking and salivary gland cancer have found no significant association [37-40]. One population-based, case-control study which included 199 salivary gland tumor cases reported that heavy drinking was associated with increased risk in men ( $\mathrm{OR}=2.5,95 \% \mathrm{CI}=1.1-5.7)$, but not in women [41]. One possible reason for this observed difference between salivary gland cancer and other HNC subtypes might be that, unlike the tissues of other HNC subtypes, the salivary glands have no direct contact with alcohol. Another possible explanation is that differences in histological type might have resulted in different relationships to alcohol; while squamous cell carcinoma is the most common pathological type in other HNC subtypes, in salivary gland cancer, adenoid cystic carcinoma and mucoepidermoid carcinoma are most common $[42,43]$. Further research with a larger number of cases is necessary to elucidate this relationship.

The most intriguing finding of our study was that drinking frequency is a more important risk factor of HNC than the daily amount of alcohol consumption. When stratified by average alcohol consumption, drinking frequency, but not daily amount, showed a linear relationship with $\mathrm{HNC}$ risk in moderate and heavy drinkers. The underlying mechanism for this finding remains unclear, but one possible explanation is that repetitive contact 
between a carcinogen (salivary AA) and the mucosa of the upper aerodigestive tract might lead these cells to increasingly undergo malignant transformations [44-47]. The direct carcinogenic or mutagenic effects of AA in saliva are usually observed at concentrations of higher than $40-50 \mu \mathrm{M}$, which can be easily reached by drinking $0.5 \mathrm{~g}$ of pure alcohol per kilogram of body weight $[45,48]$. Hence, the frequent drinking of social amount could result in prolonged exposure to the direct carcinogenic activity of AA when compared to drinking larger amounts over fewer instances. Several in vitro studies have reported that chronic, repetitive exposure to carcinogens might be required for the malignant transformation of target cells $[46,47,49]$. In a study using topical benzoapyrene (BAP), removal of BAP-DNA adducts from target tissues occurred faster after a single application than after chronic exposure, implying that repeated exposure-repair cycles of the genome promote an increase in carcinogen-DNA adducts [46]. To induce cancer development, carcinogens might need to be present in the target tissue for many years [50].

In our study, mild and moderate drinkers who drank 5-7 days/week showed a slightly lower risk of HNC compared with subjects who drank 3-4 days/week. Considering that the average amount of alcohol per occasion would have been less than $21 \mathrm{~g}$ and $42 \mathrm{~g}$, respectively, among these subjects, salivary AA concentration may have been insufficient, at least for some time, to promote local carcinogenic activity.

Our study has important clinical and public health implications. Recent guidelines on drinking habits for the prevention of cancer and chronic diseases mostly advise individuals to reduce their overall consumption or daily amount without mentioning drinking frequency [51,52]. However, there are many people who frequently drink a small amount of alcohol; for example, it is common in many cultures to have a couple of drinks with lunch or dinner. Such behavior may be reinforced in some individuals by the evidence of the preventive effect of moderate alcohol consumption on cardiovascular disease risk and all-cause mortality [53,54]. Nevertheless, recent meta-analysis and large-scale studies have suggested that even a moderate level of alcohol consumption might have no benefit regarding mortality risk $[55,56]$. As for cancer, previous research has almost consistently shown that there is no risk threshold below which alcohol drinking is not related to incidence or mortality of cancer $[8,11,57]$. In the same vein, our analysis among moderate drinkers has shown that frequent drinking of relatively low doses of alcohol can also increase the risk of HNC. The findings of our study imply that a targeted public health strategy with a higher focus on drinking frequency is needed for cancer prevention in individuals who drink a small amount frequently. While HNC is relatively rare, population-wide changes in health behaviors can induce a substantial reduction in cancer incidence [58].

Strengths of our study include being the only study to examine impacts of both drinking frequency and daily amount on HNC risk, specifically. Also, our study was based on a large, population-based, representative sample. A much higher number of HNC cases ( $n=15,832)$ were included than in previous investigations, allowing us to perform detailed analyses according to drinking patterns and cancer subtypes.

However, there are also some limitations in our study. First, due to the retrospective nature of our study, we could not obtain information on other components of drinking habits, such as the total duration of alcohol drinking or changes in drinking habits. As we were not able to exclude former drinkers who quit drinking recently, HNC risks among non-drinkers could have been exaggerated. Information on human papillomavirus infection, which is increasingly reported to increase the risk of oropharyngeal and oral cavity cancer [3], was also lacking. Also, we could not obtain data on genetic polymorphisms of alcohol-degrading enzymes. Previous studies have reported that several types of genetic polymorphisms could alter the activity of alcohol dehydrogenase and aldehyde dehydrogenase enzymes, and some of these genotypes are associated with the increases in HNC risk [59-61]. Future research might need to consider the interaction between genetic factors and alcohol drinking patterns on HNC risk. Second, information on drinking frequency or daily amount might be inaccurate because it was collected by self-reporting. Subjects might have underreported these variables or miscalculated them due to within-person variation. 
Third, since study subjects were limited to people who participated in the health screening program, the findings might be subject to selection bias: subjects might tend to engage in healthier behaviors than the general population. Hence, incidences of unhealthy lifestylessuch as drinking and smoking- might have been lower than in the general population, resulting in a lower incidence rate of HNC. Finally, as our study was limited to the Korean population, further studies are required to confirm the relationship between drinking and HNC found from our study in other populations.

\section{Conclusions}

In conclusion, this nationwide cohort study suggests that the frequency of drinking is a stronger risk factor for the development of HNC, especially for cancers of the oral cavity, pharynx, and larynx than the daily amount. Further research is needed on cancer prevention strategies with regard to drinking patterns.

Supplementary Materials: The following are available online at https://www.mdpi.com/article/ 10.3390/ijerph182111204/s1, Table S1: Risk of head \& neck cancer stratified by average alcohol consumption: Impact of drinking frequency versus daily amount, Table S2: Risks of head \& neck cancer subtypes stratified by average alcohol consumption: Impact of drinking frequency versus daily amount.

Author Contributions: Conceptualization, H.Y.K., K.H. and D.W.S.; methodology, H.Y.K., K.H., D.W.S. and J.E.Y.; formal analysis, D.K.; investigation, H.Y.K. and J.E.Y.; data curation, K.H.J. and D.K.; writing—original draft preparation, H.Y.K.; writing—review and editing, K.H., D.W.S., J.E.Y., M.H.C., S.H. and J.K.J.; visualization, H.Y.K.; supervision, D.W.S.; project administration, K.H.J; All authors have read and agreed to the published version of the manuscript.

Funding: This research received no external funding.

Institutional Review Board Statement: The study was conducted according to the guidelines of the Declaration of Helsinki and approved by the Institutional Review Board of Samsung Medical Center (IRB File No. SMC 2019-02-059).

Informed Consent Statement: The requirement for written informed consent from patients was waived because the data were anonymized under confidentiality guidelines.

Data Availability Statement: The data that support the findings of this study are available from the NHIS. Restrictions apply to the availability of these data, which were used under license for this study. Data are available from the authors with the permission of the NHIS.

Conflicts of Interest: The authors declare no conflict of interest.

\section{References}

1. Ferlay, J.; Ervik, M.; Lam, F.; Colombet, M.; Mery, L.; Piñeros, M.; Znaor, A.; Soerjomataram, I.; Bray, F. Global Cancer Observatory: Cancer Today. Lyon: International Agency for Research on Cancer 2020. Available online: https://gco.iarc.fr/today (accessed on 17 October 2021).

2. Gupta, B.; Johnson, N.W.; Kumar, N. Global Epidemiology of Head and Neck Cancers: A Continuing Challenge. Oncology 2016, 91, 13-23. [CrossRef]

3. Shaw, R.; Beasley, N. Aetiology and risk factors for head and neck cancer: United Kingdom National Multidisciplinary Guidelines. J. Laryngol. Otol. 2016, 130 (Suppl. 2), S9-S12. [CrossRef]

4. Dhull, A.K.; Atri, R.; Dhankhar, R.; Chauhan, A.K.; Kaushal, V. Major Risk Factors in Head and Neck Cancer: A Retrospective Analysis of 12-Year Experiences. World J. Oncol. 2018, 9, 80-84. [CrossRef]

5. Freedman, N.D.; Schatzkin, A.; Leitzmann, M.F.; Hollenbeck, A.R.; Abnet, C. Alcohol and head and neck cancer risk in a prospective study. Br. J. Cancer 2007, 96, 1469-1474. [CrossRef]

6. Polesel, J.; Maso, L.D.; Bagnardi, V.; Zucchetto, A.; Zambon, A.; Levi, F.; La Vecchia, C.; Franceschi, S. Estimating dose-response relationship between ethanol and risk of cancer using regression spline models. Int. J. Cancer 2005, 114, 836-841. [CrossRef]

7. Zhang, Y.; Wang, R.; Miao, L.; Zhu, L.; Jiang, H.; Yuan, H. Different Levels in Alcohol and Tobacco Consumption in Head and Neck Cancer Patients from 1957 to 2013. PLoS ONE 2015, 10, e0124045. [CrossRef] [PubMed]

8. Bagnardi, V.; Blangiardo, M.; la Vecchia, C.; Corrao, G. Alcohol consumption and the risk of cancer: A meta-analysis. Alcohol Res. Health 2001, 25, 263-270. 
9. Mørch, L.S.; Johansen, D.; Thygesen, L.; Tjonneland, A.; Løkkegaard, E.; Stahlberg, C.; Grønbaek, M. Alcohol drinking, consumption patterns and breast cancer among Danish nurses: A cohort study. Eur. J. Public Health 2007, 17, 624-629. [CrossRef] [PubMed]

10. Platz, E.A.; Leitzmann, M.F.; Rimm, E.B.; Willett, W.C.; Giovannucci, E. Alcohol Intake, Drinking Patterns, and Risk of Prostate Cancer in a Large Prospective Cohort Study. Am. J. Epidemiol. 2004, 159, 444-453. [CrossRef] [PubMed]

11. Cao, Y.; Willett, W.C.; Rimm, E.B.; Stampfer, M.J.; Giovannucci, E.L. Light to moderate intake of alcohol, drinking patterns, and risk of cancer: Results from two prospective US cohort studies. BMJ 2015, 351, h4238. [CrossRef] [PubMed]

12. Kjærheim, K.; Gaard, M.; Andersen, A. The role of alcohol, tobacco, and dietary factors in upper aerogastric tract cancers: A prospective study of 10,900 Norwegian men. Cancer Causes Control. 1998, 9, 99-108. [CrossRef]

13. Di Credico, G.; Polesel, J.; Maso, L.D.; Pauli, F.; Torelli, N.; Luce, D.; Radoï, L.; Matsuo, K.; Serraino, D.; Brennan, P.; et al. Alcohol drinking and head and neck cancer risk: The joint effect of intensity and duration. Br. J. Cancer 2020, 123, 1456-1463. [CrossRef]

14. Gupta, B.; Kumar, N.; Johnson, N.W. Relationship of Lifetime Exposure to Tobacco, Alcohol and Second Hand Tobacco Smoke with Upper aero-digestive tract cancers in India: A Case-Control Study with a Life-Course Perspective. Asian Pac. J. Cancer Prev. 2017, 18, 347-356.

15. Gupta, B.; Bray, F.; Kumar, N.; Johnson, N.W. Associations between oral hygiene habits, diet, tobacco and alcohol and risk of oral cancer: A case-control study from India. Cancer Epidemiol. 2017, 51, 7-14. [CrossRef]

16. Du, T.; Chen, K.; Zheng, S.; Bao, M.; Huang, Y.; Wu, K. Association between Alcohol Consumption and Risk of Nasopharyngeal Carcinoma: A Comprehensive Meta-Analysis of Epidemiological Studies. Alcohol Clin. Exp. Res. 2019, 43, 2262-2273. [CrossRef]

17. Seong, S.C.; Kim, Y.-Y.; Park, S.K.; Khang, Y.-H.; Kim, H.C.; Park, J.H.; Kang, H.-J.; Do, C.-H.; Song, J.-S.; Lee, E.-J.; et al. Cohort profile: The National Health Insurance Service-National Health Screening Cohort (NHIS-HEALS) in Korea. BMJ Open 2017, 7, e016640. [CrossRef]

18. Kalinowski, A.; Humphreys, K. Governmental standard drink definitions and low-risk alcohol consumption guidelines in 37 countries. Addiction 2016, 111, 1293-1298. [CrossRef]

19. Furtwaengler, N.A.; de Visser, R.O. Lack of international consensus in low-risk drinking guidelines. Drug Alcohol Rev. 2013, 32, 11-18. [CrossRef]

20. Kim, Y.G.; Han, K.; Choi, J., II; Boo, K.Y.; Kim, D.Y.; Lee, K.; Shim, J.; Kim, J.S.; Kim, Y.-H. Frequent drinking is a more important risk factor for new-onset atrial fibrillation than binge drinking: A nationwide population-based study. Europace 2020, 22, 216-224. [CrossRef]

21. Korea Health Promotion Institute. Guideline for Low-Risk Alcohol Consumption. 2013. Available online: https://www.khealth or.kr/kps/publish/view?menuId=MENU00891\&page_no=B2017004\&board_idx=7640 (accessed on 5 August 2021).

22. Stott-Miller, M.; Chen, C.; Chuang, S.-C.; Lee, Y.-C.A.; Boccia, S.; Brenner, H.; Cadoni, G.; Maso, L.D.; La Vecchia, C.; Lazarus, P.; et al. History of diabetes and risk of head and neck cancer: A pooled analysis from the international head and neck cancer epidemiology consortium. Cancer Epidemiol. Biomark. Prev. 2012, 21, 294-304. [CrossRef]

23. Tseng, K.S.; Lin, C.; Lin, Y.-S.; Weng, S.-F. Risk of head and neck cancer in patients with diabetes mellitus: A retrospective cohort study in Taiwan. JAMA Otolaryngol. Head Neck Surg. 2014, 140, 746-753. [CrossRef]

24. Seo, J.-H.; Kim, Y.-D.; Park, C.-S.; Han, K.-D.; Joo, Y.-H. Hypertension is associated with oral, laryngeal, and esophageal cancer: A nationwide population-based study. Sci. Rep. 2020, 10, 10291. [CrossRef]

25. Kao, L.-T.; Hung, S.-H.; Kao, P.-F.; Liu, J.-C.; Lin, H.-C. Inverse association between statin use and head and neck cancer: Population-based case-control study in Han population. Head Neck 2019, 41, 1193-1198. [CrossRef]

26. Maasland, D.; van den Brandt, P.A.; Kremer, B.; Goldbohm, R.A.S.; Schouten, L.J. Alcohol consumption, cigarette smoking and the risk of subtypes of head-neck cancer: Results from the Netherlands Cohort Study. BMC Cancer 2014, 14, 187. [CrossRef]

27. Grønbaek, M.; Becker, U.; Johansen, D.; Tønnesen, H.; Jensen, G.; Sørensen, T.I. Population based cohort study of the association between alcohol intake and cancer of the upper digestive tract. BMJ 1998, 317, 844-847. [CrossRef]

28. Goldstein, B.Y.; Chang, S.-C.; Hashibe, M.; La Vecchia, C.; Zhang, Z.-F. Alcohol consumption and cancers of the oral cavity and pharynx from 1988 to 2009: An update. Eur. J. Cancer Prev. 2010, 19, 431-465. [CrossRef]

29. Seitz, H.K.; Becker, P. Alcohol metabolism and cancer risk. Alcohol Res. Health 2007, 30, 38-47.

30. Seitz, H.K.; Stickel, F.; Homann, N. Pathogenetic mechanisms of upper aerodigestive tract cancer in alcoholics. Int. J. Cancer 2004, 108, 483-487. [CrossRef]

31. Obe, G.; Jonas, R.; Schmidt, S. Metabolism of ethanol in vitro produces a compound which induces sister-chromatid exchanges in human peripheral lymphocytes in vitro: Acetaldehyde not ethanol is mutagenic. Mutat. Res. Lett. 1986, 174, 47-51. [CrossRef]

32. Yokoyama, A.; Tsutsumi, E.; Imazeki, H.; Suwa, Y.; Nakamura, C.; Mizukami, T.; Yokoyama, T. Salivary acetaldehyde concentration according to alcoholic beverage consumed and aldehyde dehydrogenase-2 genotype. Alcohol Clin. Exp. Res. 2008, 32, 1607-1614. [CrossRef]

33. Lachenmeier, D.W.; Monakhova, Y.B. Short-term salivary acetaldehyde increase due to direct exposure to alcoholic beverages as an additional cancer risk factor beyond ethanol metabolism. J. Exp. Clin. Cancer Res. 2011, 30, 3. [CrossRef] [PubMed]

34. Mizumoto, A.; Ohashi, S.; Hirohashi, K.; Amanuma, Y.; Matsuda, T.; Muto, M. Molecular Mechanisms of Acetaldehyde-Mediated Carcinogenesis in Squamous Epithelium. Int. J. Mol. Sci. 2017, 18, 1943. [CrossRef] [PubMed]

35. Stornetta, A.; Guidolin, V.; Balbo, S. Alcohol-Derived Acetaldehyde Exposure in the Oral Cavity. Cancers 2018, 10, 20. [CrossRef] [PubMed] 
36. Filion, E.J.; McClure, L.A.; Huang, D.; Seng, K.; Kaplan, M.J.; Colevas, A.D.; Gomez, S.L.; Chang, E.T.; Le, Q.-T. Higher incidence of head and neck cancers among Vietnamese American men in California. Head Neck 2010, 32, 1336-1344. [CrossRef]

37. Pan, S.Y.; de Groh, M.; Morrison, H. A Case-Control Study of Risk Factors for Salivary Gland Cancer in Canada. J. Cancer Epidemiol. 2017, 2017, 4909214. [CrossRef]

38. Zheng, W.; Shu, X.-O.; Ji, B.-T.; Gao, Y.-T. Diet and other risk factors for cancer of the salivary glands:a population-based case-control study. Int. J. Cancer 1996, 67, 194-198. [CrossRef]

39. Muscat, J.E.; Wynder, E.L. A case/control study of risk factors for major salivary gland cancer. Otolaryngol. Head Neck Surg. 1998, 118, 195-198. [CrossRef]

40. Hayes, R.; Bravo-Otero, E.; Kleinman, D.V.; Brown, L.M.; Fraumeni, J.F.; Harty, L.C.; Winn, D.M. Tobacco and alcohol use and oral cancer in Puerto Rico. Cancer Causes Control 1999, 10, 27-33. [CrossRef]

41. Horn-Ross, P.L.; Ljung, B.-M.; Morrow, M. Environmental Factors and the Risk of Salivary Gland Cancer. Epidemiology 1997, 8, 414-419. [CrossRef]

42. Sanderson, R.J.; Ironside, J.A. Squamous cell carcinomas of the head and neck. BMJ 2002, 325, 822-827. [CrossRef]

43. Wang, Y.-L.; Zhu, Y.-X.; Chen, T.-Z.; Wang, Y.; Sun, G.-H.; Zhang, L.; Huang, C.-P.; Wang, Z.-Y.; Shen, Q.; Li, D.-S.; et al. Clinicopathologic study of 1176 salivary gland tumors in a Chinese population: Experience of one cancer center 1997-2007. Acta Otolaryngol. 2012, 132, 879-886. [CrossRef]

44. Väkeväinen, S.; Tillonen, J.; Agarwal, D.P.; Srivastava, N.; Salaspuro, M. High Salivary Acetaldehyde After a Moderate Dose of Alcohol in ALDH2-Deficient Subjects: Strong Evidence for the Local Carcinogenic Action of Acetaldehyde. Alcohol. Clin. Exp. Res. 2000, 24, 873-877. [CrossRef]

45. Homann, N.; Jousimies-Somer, H.; Jokelainen, K.; Heine, R.; Salaspuro, M. High acetaldehyde levels in saliva after ethanol consumption: Methodological aspects and pathogenetic implications. Carcinogenesis 1997, 18, 1739-1743. [CrossRef]

46. Talaska, G.; Jaeger, M.; Reilman, R.; Collins, T.; Warshawsky, D. Chronic, topical exposure to benzo[a]pyrene induces relatively high steady-state levels of DNA adducts in target tissues and alters kinetics of adduct loss. Proc. Natl. Acad. Sci. USA 1996, 93, 7789-7793. [CrossRef]

47. Mennecier, G.; Torres, L.N.; Cogliati, B.; Sanches, D.S.; Mori, C.M.; Latorre, A.O.; Chaible, L.M.; Mackowiak, I.I.; Nagamine, M.K.; Da Silva, T.C.; et al. Chronic exposure of lung alveolar epithelial type II cells to tobacco-specific carcinogen NNK results in malignant transformation: A new in vitro lung carcinogenesis model. Mol. Carcinog. 2014, 53, 392-402. [CrossRef]

48. Sarkola, T.; Iles, M.R.; Kohlenberg-Mueller, K.; Eriksson, C.J.P. Ethanol, acetaldehyde, acetate, and lactate levels after alcohol intake in white men and women: Effect of 4-methylpyrazole. Alcohol Clin. Exp. Res. 2002, 26, 239-245. [CrossRef]

49. Belinsky, S.A.; Devereux, T.R.; Foley, J.F.; Maronpot, R.R.; Anderson, M.W. Role of the Alveor Type II Cell in the Development and Progression of Pulmonary Tumors Induced bt 4-(Methylnitrosamino)-(3-pyridyl)-1-butanone in the A/J Mouse. Cancer Res. 1992, 52, 3164-3173.

50. Oliveira, P.A.; Colaço, A.; Chaves, R.; Guedes-Pinto, H.; De-La-Cruz, P.L.F.; Lopes, C. Chemical carcinogenesis. An. Acad. Bras. Cienc. 2007, 79, 593-616. [CrossRef]

51. Rock, C.L.; Thomson, C.; Gansler, T.; Gapstur, S.M.; McCullough, M.L.; Patel, A.V.; Ba, K.S.A.; Bandera, E.V.; Spees, C.K.; Robien, K.; et al. American Cancer Society guideline for diet and physical activity for cancer prevention. CA Cancer J. Clin. 2020, 70, 245-271. [CrossRef]

52. Willenbring, M.L.; Massey, S.H.; Gardner, M.B. Helping patients who drink too much: An evidence-based guide for primary care clinicians. Am. Fam. Physician 2009, 80, 44-50.

53. Kannel, W.B.; Ellison, R.C. Alcohol and coronary heart disease: The evidence for a protective effect. Clin. Chim. Acta 1996, 246, 59-76. [CrossRef]

54. Di Castelnuovo, A.; Costanzo, S.; Bagnardi, V.; Donati, M.B.; Iacoviello, L.; de Gaetano, G. Alcohol dosing and total mortality in men and women: An updated meta-analysis of 34 prospective studies. Arch. Intern. Med. 2006, 166, 2437-2445. [CrossRef]

55. Stockwell, T.; Zhao, J.; Panwar, S.; Roemer, A.; Naimi, T.; Chikritzhs, T. Do "Moderate” Drinkers Have Reduced Mortality Risk? A Systematic Review and Meta-Analysis of Alcohol Consumption and All-Cause Mortality. J. Stud. Alcohol Drugs 2016, 77, 185-198. [CrossRef]

56. Goulden, R. Moderate Alcohol Consumption Is Not Associated with Reduced All-cause Mortality. Am. J. Med. 2016, 129, 180-186.e4. [CrossRef]

57. Ko, H.; Chang, Y.; Kim, H.-N.; Kang, J.-H.; Shin, H.; Sung, E.; Ryu, S. Low-level alcohol consumption and cancer mortality. Sci. Rep. 2021, 11, 4585. [CrossRef]

58. Institute of Medicine and Board National Research Council National Cancer Policy. Fulfilling the Potential of Cancer Prevention and Early Detection; Curry, S.J., Byers, T., Hewitt, M., Eds.; National Academies Press: Washington, DC, USA, 2003; Copyright 2003 by the National Academy of Sciences.

59. Brennan, P.; Lewis, S.; Hashibe, M.; Bell, D.A.; Boffetta, P.; Bouchardy, C.; Caporaso, N.; Chen, C.; Coutelle, C.; Diehl, S.R.; et al. Pooled analysis of alcohol dehydrogenase genotypes and head and neck cancer: A HuGE review. Am. J. Epidemiol. 2004, 159, 1-16. [CrossRef]

60. Kawakita, D.; Matsuo, K. Alcohol and head and neck cancer. Cancer Metastasis Rev. 2017, 36, 425-434. [CrossRef]

61. Chien, H.-T.; Young, C.-K.; Chen, T.-P.; Liao, C.-T.; Wang, H.-M.; Cheng, S.-D.; Huang, S.-F. Alcohol-metabolizing Enzymes' Gene Polymorphisms and Susceptibility to Multiple Head and Neck Cancers. Cancer Prev. Res. 2019, 12, 247-254. [CrossRef] 